\title{
Small Bowel Angiodysplasia Managed with Novel Motorized Spiral Enteroscope: A Case Report
}

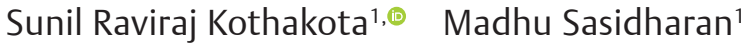 \\ ${ }^{1}$ Department of Gastroenterology, Kerala Institute of Medical \\ Sciences Hospital, Trivandrum, Kerala, India
}

\author{
Harish Kareem ${ }^{1} \quad$ Ajith K. Nair ${ }^{1}$
}

\begin{abstract}
Address for correspondence Sunil Raviraj Kothakota, MBBS, DNB, Department of Gastroenterology, Kerala Institute of Medical Sciences Hospital, PO Box Anayara, Trivandrum 695029, Kerala, India (e-mail: ksrdnb@gmail.com).
\end{abstract}

\begin{abstract}
Keywords

- small bowel bleed

- ileal angiodysplasia

- spiral enteroscope

We report a case of 72-year-old woman, who presented with complaints of melena associated with shortness of breath on exertion for more than 2 weeks. On evaluation, she was diagnosed to have severe anemia with evidence of occult blood in stool. After initial management, further workup was done to find out the source of bleeding. Upper and lower gastrointestinal endoscopy were normal. Computerized tomography enteroclysis showed no significant abnormality. Later small bowel enteroscopy was performed with the help of Novel Motorized Spiral Enteroscope in antegrade approach and scope passed through oral cavity up to cecum. Multiple angiodysplasia lesions seen in ileum. All the lesions were managed with argon plasma coagulation (APC). Postprocedure, patient improved well and hemoglobin also remained stable. Small bowel diseases always remain a diagnostic and therapeutic challenge, despite the introduction of various modalities for deep enteroscopy. Spiral enteroscopy is a recent advance which uses an overtube with a raised spiral at the distal end to pleat the small intestine. It consumes less time and suits for both diagnostic and therapeutic needs of small bowel diseases.
\end{abstract}

\section{Introduction}

The push-and-pull enteroscopy, double balloon and single balloon enteroscopy (DBE and SBE, respectively), ${ }^{1,2}$ was introduced for the visualization of small bowel diseases, however, they are very time consuming. Availability of video capsule enteroscopy (VCE) permits the diagnostic study of small bowel but limited by lacking the therapeutic role.

Spiral enteroscopy (SE) is a new technique that uses an overtube with raised spiral at distal end, which is time conserving and suits for both diagnostic and therapeutic needs. ${ }^{3}$ Initial model of SE works by manual clockwise rotation of spiral overtube for inducing pleating of the small bowel on the endoscope. The Novel Motorized Spiral Enteroscope (NMSE, Olympus Corp.) is a new technology with an incorporated user-controlled motor contained in the handle of the endoscope. The power spiral control unit controls the rotational direction and speed of the spiral segment, which is displayed visually for monitoring on a force gauge. The gentle grip on the mucosa between the soft fins of the spiral segment enables precise positioning of the endoscope and integrated water jet keeps the view clear. As it is not widely in use, the available literature regarding the spiral enteroscopy is scanty. We managed a case of elderly woman with small bowel (occult) bleed with this novel modality.

\section{Case Summary}

A 72-year-old woman, with a history of diabetes mellitus, presented with complaints of melena associated with breathlessness on exertion for more than 2 weeks. Clinical survey was normal except for presence of pallor. On workup, she was found to have low hemoglobin $(6.5 \mathrm{~g} \%)$ and stool occult blood was positive. Renal and hepatic parameters were within normal limits. Anemia profile was suggestive of iron-deficiency anemia. She was managed with packed red blood cells (PRBCs) along with other supportive measures. Upper gastrointestinal endoscopy and ileocolonoscopy were 


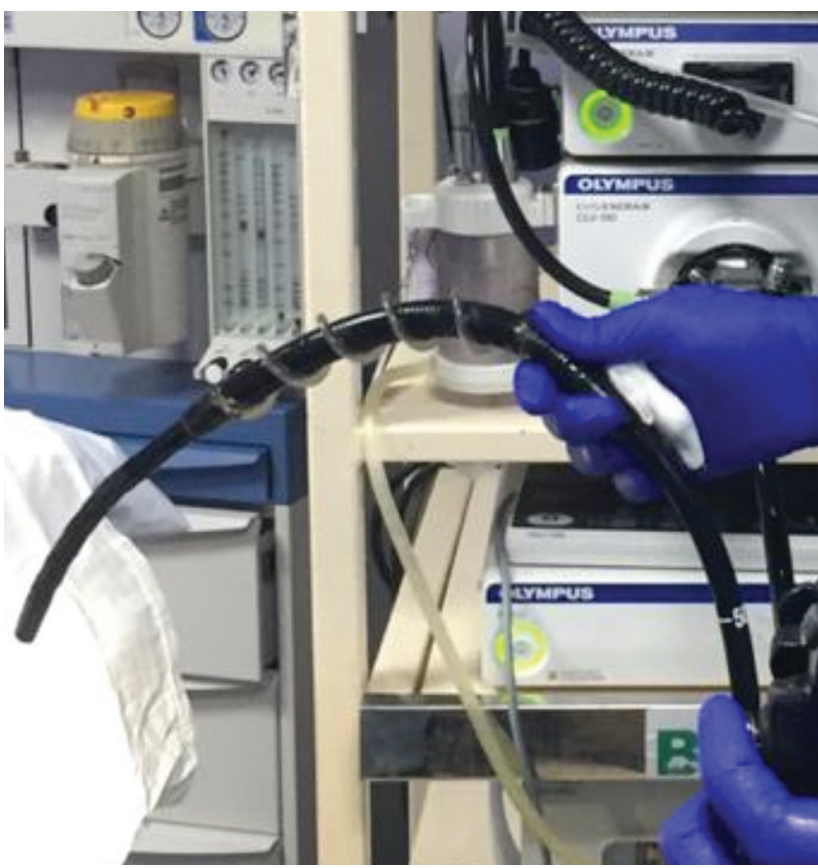

Fig. 1 Novel Motorized Spiral Enteroscope (NMSE; Olympus corp.) with spiral overtube at distal end.

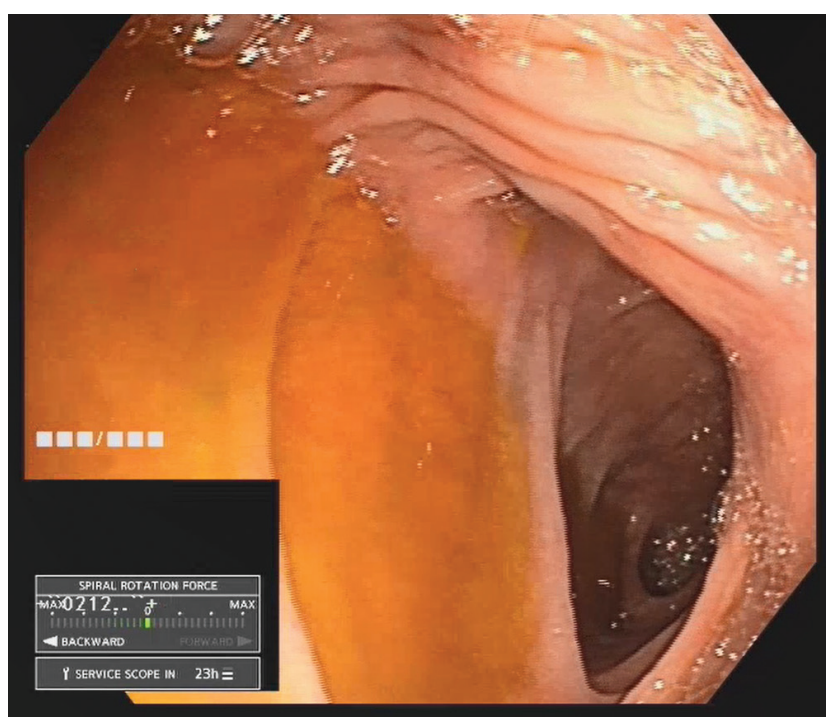

Fig. 2 Antegrade view of cecum through ileum.

done and both were unremarkable. Ultrasound abdomen and computerized tomography (CT) enteroclysis were also normal. Later small bowel evaluation was performed by using NMSE ( - Fig. 1) under monitored anesthesia care. Patient was kept on left lateral posture, scope introduced through oral cavity, and complete small bowel study was done, scope progressed up to cecum within 24 minutes ( $\boldsymbol{\sim}$ Fig. 2). Duodenum and jejunum were normal. Multiple angiodysplasia lesions ( - Fig. 3) were noted in proximal ileum and applied argon plasma coagulation (APC) to all lesions during the same session (-Fig. 4). Postprocedure patient recovered well. She was started on thalidomide and discharged. During 3 month postprocedure follow-up period, she never had recurrence of symptoms and her hemoglobin level remained stable.

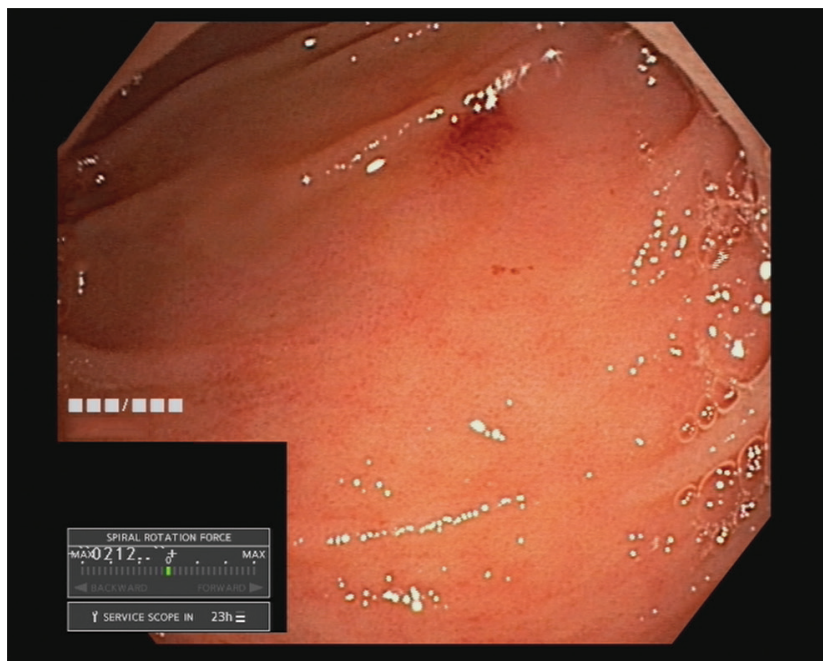

Fig. 3 Angiodysplasia lesion in ileum.

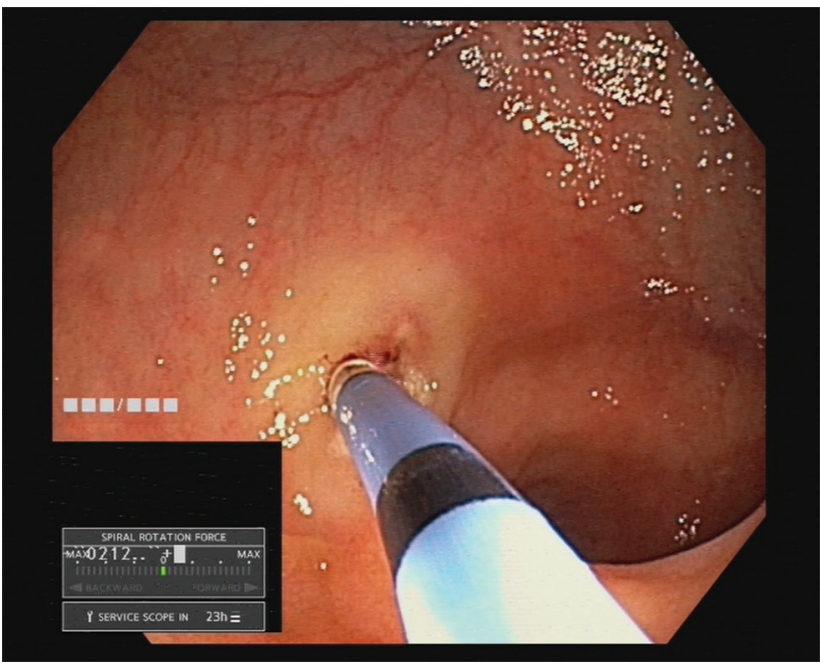

Fig. 4 Argon plasma coagulation for angiodysplasia lesions.

\section{Discussion}

Small bowel pathologies always remains as a challenge because of lack of accurate endoscopic modalities. During the last two decades, the invention of DBE in 2001 and SBE in 2006 enable the deep enteroscopy ${ }^{12}$; however both are time consuming. Spiral enteroscopy is an alternate method for small bowel study in which a special overtube called the discovery small bowel (DSB) with raised helices at the distal end is equipped. Clockwise rotation of the DSB pleats the small bowel on to the overtube. This prototype model needs additional manpower during the procedure to maintain the DSB rotation manually. NMSE is the newer development with same principle, equipped with integrated motor for rotating a disposable short spiral overtube mounted on the insertion tube. ${ }^{4}$ This novel instrument is of $160 \mathrm{~cm}$ length with $3.2 \mathrm{~mm}$ accessory channel, similar to standard colonoscope. The literature on spiral enteroscopy is still developing. It offers diagnostic and therapeutic capabilities comparable with other modalities while consuming less time. ${ }^{5}$ In our study, we could reach the cecum from oral cavity within 
24 minutes. The well-controlled movement of the scope tip and counter-clock rotation of overtube (for unpleat the bowel) during withdrawal allowed us to visualize all the angiodysplasia lesions ( - Fig. 3) and to manage them with APC (-Fig. 4). Complications like pancreatitis and perforation were reported with the use of spiral enteroscope ${ }^{6}$; however in our case, the study was uneventful. Further largescale studies are needed to assess the diagnostic yield and complications of this procedure.

\section{Financial Support and Sponsorship}

Nil.

\section{Conflicts of Interest}

None.

\section{References}

1 Yamamoto $\mathrm{H}$, Sekine Y, Sato Y, et al. Total enteroscopy with a nonsurgical steerable double-balloon method. Gastrointest Endosc 2001;53(2):216-220
2 Tsujikawa T, Saitoh Y, Andoh A, et al. Novel single-balloon enteroscopy for diagnosis and treatment of the small intestine: preliminary experiences. Endoscopy 2008;40(1):11-15

3 Akerman PA, Agrawal D, Chen W, Cantero D, Avila J, Pangtay J. Spiral enteroscopy: a novel method of enteroscopy by using the endo-ease discovery SB overtube and a pediatric colonoscope. Gastrointest Endosc 2009;69(2):327-332

4 Neuhaus H, Beyna T, Schneider M, Devière J. Novel motorized spiral enteroscopy: first clinical case. VideoGIE 2016;1(2): 32-33

5 May A, Manner H, Aschmoneit I, Ell C. Prospective, cross-over, single-center trial comparing oral double-balloon enteroscopy and oral spiral enteroscopy in patients with suspected small-bowel vascular malformations. Endoscopy 2011;43(6): 477-483

6 Akerman PA, Agrawal D, Cantero D, Pangtay J. Spiral enteroscopy with the new DSB overtube: a novel technique for deep peroral small-bowel intubation. Endoscopy 2008;40(12): 974-978 